\title{
Structure of the fully modified left-handed cyclohexene nucleic acid sequence GTGTACAC
}

by

Koen Robeyns, Piet Herdewijn and Luc Van Meervelt

\section{SUPPLEMENTARY MATERIAL}
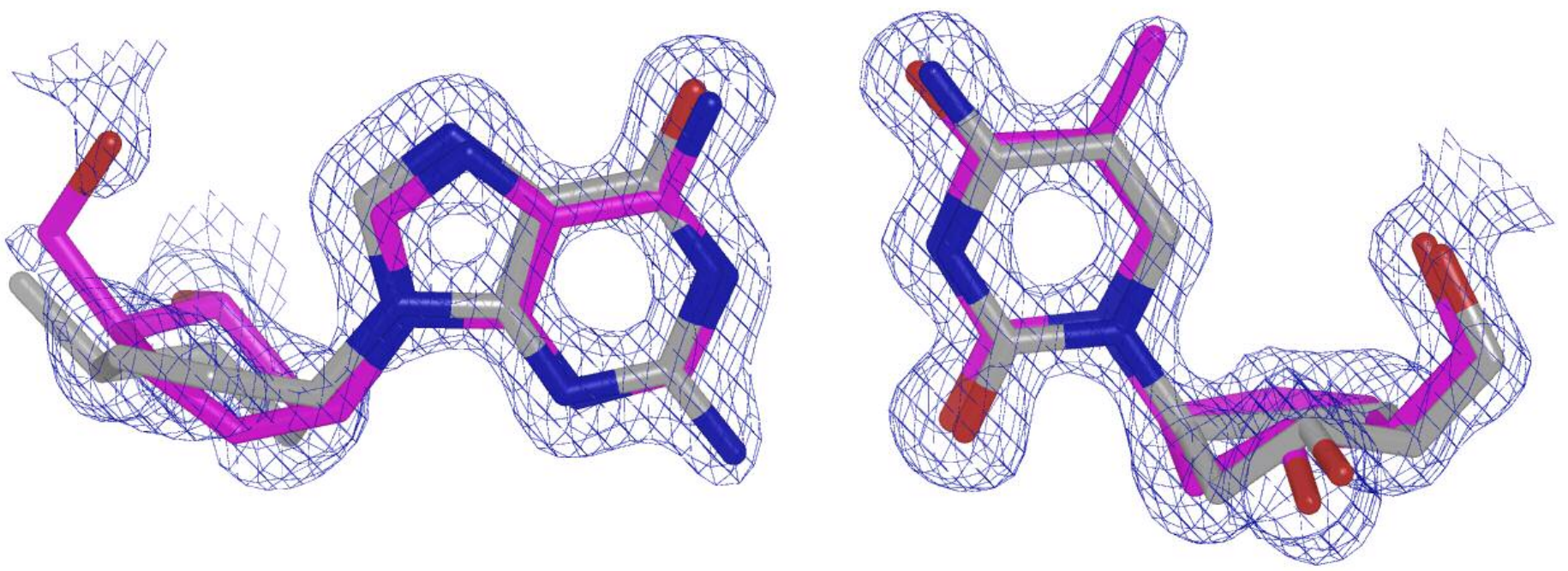

a)
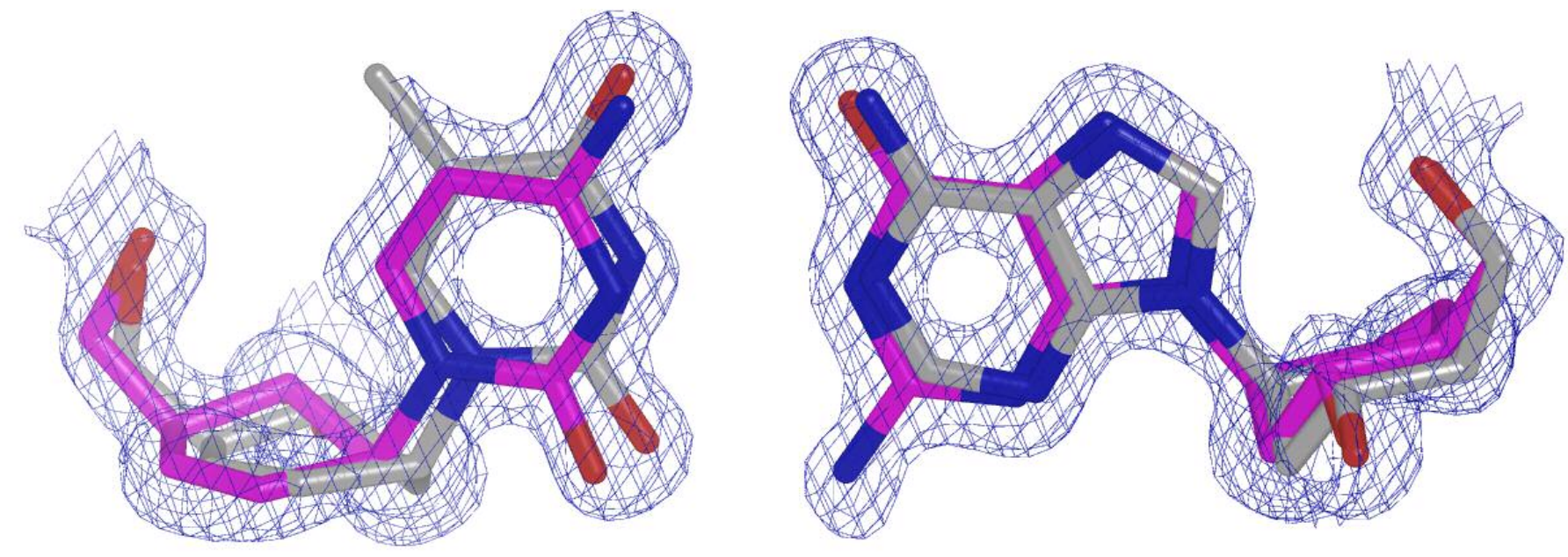

b) 

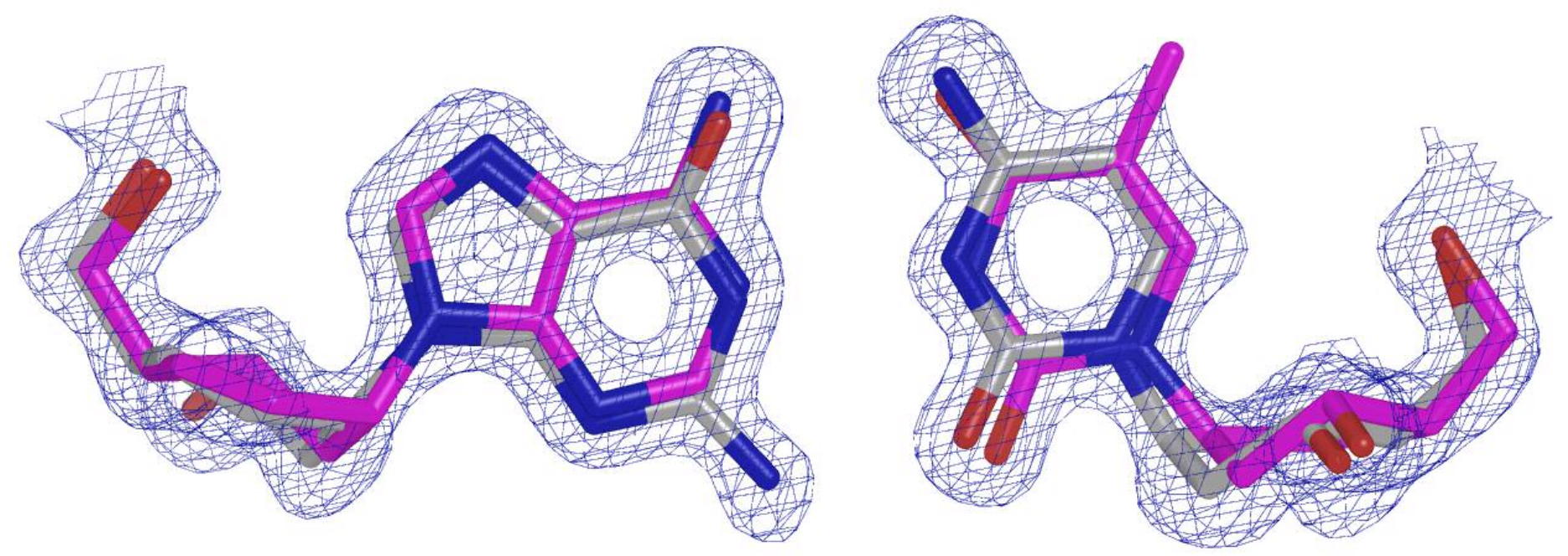

c)
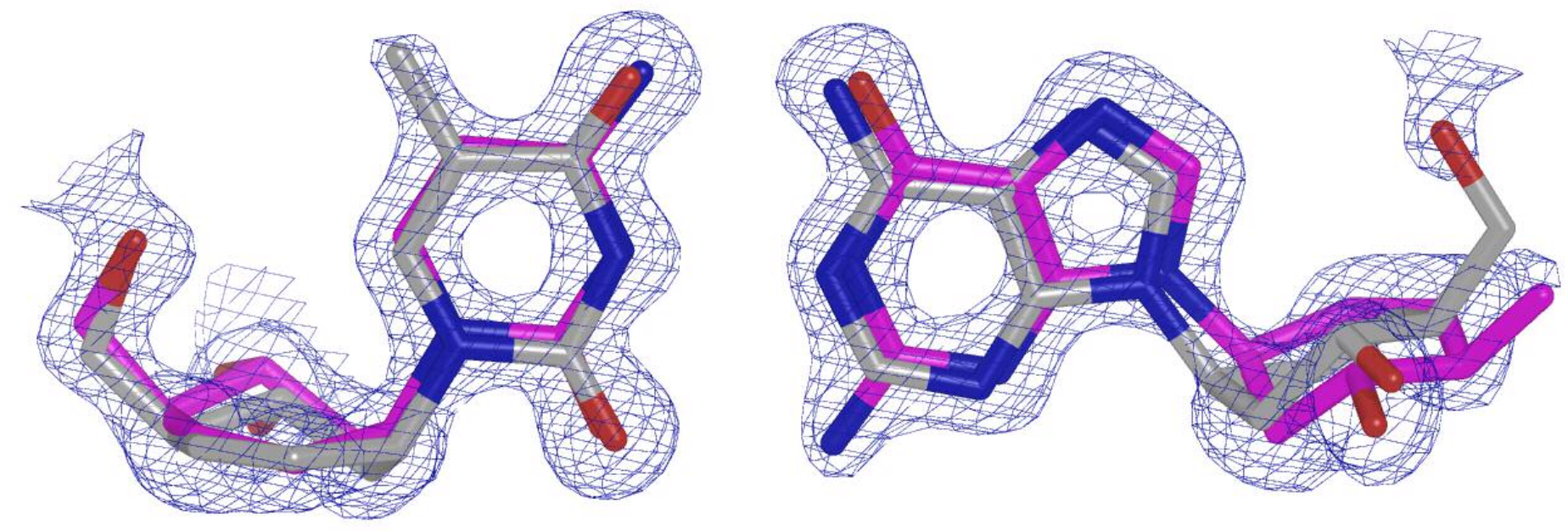

d)

Figure S1. $2\left|\mathrm{~F}_{0}\right|-\left|\mathrm{F}_{\mathrm{c}}\right|$ electron density maps contoured at the $1.1 \sigma$ level for the individual base pairs present in the crystal structure of the CeNA sequence GTGTACAC. Due to static disorder two parts are observed. Part one is shifted four base pairs relative to part two. The sugar phosphate backbone occupies similar positions for both parts. A.T base pairs of part two are superposed onto G.C base pairs of part one and vice versa. Figure a to d show the individual base pairs 1 to 4 of part one (in grey)together with the relevant base pairs of part two (in magenta) superposed onto the electron density map. 